\title{
Chirality pairing recognition, a unique reaction forming spiral alkaloids from amino acids stereoselectively in one-pot
}

\author{
Bing BAI, ${ }^{\mathrm{a}, \mathrm{c}}$ Da-Shan LI, ${ }^{\mathrm{a}}$ Sheng-Zhuo HuANG, ${ }^{\mathrm{a}} \mathrm{Jie}$ ReN,,${ }^{\mathrm{a}, \mathrm{b}}$ and Hua-Jie Zhu ${ }^{\mathrm{a}, \mathrm{b}, *}$ \\ ${ }^{a}$ State Key Laboratory of Phytochemistry and Plant Resources in West China, Kunming Institute of Botany, Chinese \\ Academy of Sciences, Kunming 650201, China \\ ${ }^{b}$ Key Laboratory of Medicinal Chemistry and Molecular Diagnosis of Ministry of Education, College of Life Science, Hebei \\ University, Baoding, 071002, China \\ ${ }^{\mathrm{c}}$ Graduate University of Chinese Academy of Sciences, Beijing 100049, China
}

Received 10 January 2012; Accepted 21 February 2012

(C) The Author(s) 2012. This article is published with open access at Springerlink.com

\begin{abstract}
A novel chirality pairing recognition was found between D- and L-amino acid derivatives. Novel spiral alkaloids formed in the recognition reaction. Possible mechanism was proposed for the stereoselective and chemoselective reactions.
\end{abstract}

Keywords: chirality pairing recognition reaction, spiral alkaloid, amino acid derivatives

\section{Introduction}

Sexual selectivity and recognition are the important characteristics of life in evolutions in nature since "sex" means re-combination between two "sexual units", and "recognition" means one unit can select the most suitable one for its re-productions, which looks like a male needs a female in a family. Although "molecular sex" was first used in nucleic acids hybridize studies", and "molecular recognition" has been widely used in chiral compound separations, which via space match each other, however, they are physical behaviors. ${ }^{2}$ In this report, we show the first chemical behavior example of chirality pairing recognition reaction or molecular sex recognition reactions where $\mathrm{D}-(+)$-amino acid derivative could selectively react with the corresponding L-(-)-amino acid analog to form single product combined by $\mathrm{D}$ - and $\mathrm{L}$-amino derivative (D-L) instead of mixtures of D-D and L-L products. Novel spiral alkaloid with unique sketch formed in the one-pot reactions.

\section{Results and Discussion}

Our initial attempt at synthesizing chiral compound 3 by reacting an oxalaldehyde with a tryptophan methyl ester (1) in $\mathrm{CH}_{2} \mathrm{Cl}_{2}$ did not yield the desired product ${ }^{3}$ (Fig. 1). The major compound (60\% yield) obtained had neither the quaternary C-4'a at $108.3 \mathrm{ppm}$ nor C-9'a at $130.8 \mathrm{ppm}$, but had one new quaternary ${ }^{13} \mathrm{C}$ at 64.7 and one new tertiary ${ }^{13} \mathrm{C}$ at $88.3 \mathrm{ppm}$. This indicated that one $\mathrm{C}=\mathrm{C}$ of the second indole moiety

\footnotetext{
*To whom correspondence should be addressed. E-mail:
} zhuhuajie@hotmail.com became a $\mathrm{C}-\mathrm{C}$ bond. A new condensation reaction happened in the procedure after normal Pictet-Spengler reaction in the first condensations. ${ }^{4} \mathrm{H}-\mathrm{H}$ COSY, HMBC and HSQC experimental results exhibited that its planar structure as 4 . The key NOE between the $\mathrm{H}-3 / \mathrm{H}-9^{\prime}$ and $\mathrm{H}-1 / \mathrm{H}-\mathrm{l}^{\prime}$ in ROESY experiments suggested that the major product have the 4a stereochemistry. Moreover, the experimental high resolution MS data (459.2022, $[\mathrm{M}+\mathrm{H}]^{+}$) agrees well with the calculated $\mathrm{M}^{+}$of 458.1954 (Fig. 1). Similarly, the minor product had the structure of $\mathbf{4 b}$ from $c i s-2$.

We also used amines $(\mathbf{5}, \mathbf{6}, 9$ and $\mathbf{1 0})$ and esters $(\mathbf{7}$ and $\mathbf{8})$ in the study. When $\mathbf{5}$ was used as the starting material, compound 11 were obtained with about $47 \%$ yield. Similarly, 12 from 6, 13 from 7, 14 from 8 and 15 from 9 were obtained, respectively. The results are summarized below. It was found that the presence of a strong electron-withdrawing group of $-\mathrm{OH}$ on the indole ring of $\mathbf{1 0}$ inhibited the reaction to $\mathbf{1 6}$, while the presence of $\mathrm{CH}_{3}$ on 9 promoted the reaction to $\mathbf{1 5}$.

A mechanism for the transformation is proposed (Fig. 2) whereby after the second Schiff base (17) is formed, N-2' can chelate with an $\mathrm{H}^{+}$to form the first five-membered ring (C-1'C-4'a formation, 18), then the positive charge can transfer to C-9'a. N-2 in 19 immediately connected to C-9'a after loss of a $\mathrm{H}^{+}$via a concerned procedure. ${ }^{5}$ The key requirement in the cycloaddition reactions is that a proton must be present on the indole $N$ atom. Thus, if this proton was replaced by a methyl group as in $\mathbf{2 0}$ and $\mathbf{2 1}$, the condensation cannot take place. This prediction is recorded when $\mathbf{2 0}$ or $\mathbf{2 1}$ was used in the condensations. 
<smiles>COC(=O)[C@H](N)Cc1c[nH]c2ccccc12</smiles><smiles>COC(=O)C1Cc2c([nH]c3ccccc23)C(C2NC(C(=O)OC)C(C(=O)OC)Cc3c2[nH]c2ccccc32)N1</smiles>

Fig. 1. Possible structures in the new condensation reactions

The unexpected molecular chirality pairing recognition was observed when a (D)-amino acid derivative was mixed with an equal molar of the corresponding (L)-amino acid derivative. For example, D-(+)-1 reacted with L-(-)-1, only one major product formed. This product can be separated as two compounds using chiral column. Their ${ }^{1} \mathrm{H}$ and ${ }^{13} \mathrm{C}$ NMR were the same, and the determined optical rotation for $\mathbf{2 2}$ was +187.3 , while the ent-22 had -189.2 in chloroform. It exhibited that the separated two products were the enantiomers. Its planar structure was well established as 22 using ${ }^{13} \mathrm{C}$ NMR and HMBC spectra (see Electronic Supplementary Material for more details). Its relative configuration was identified using ROESY experiments. The key interactions between the $\mathrm{H}-3$ and $\mathrm{H}-9$ 'a, and $\mathrm{H}-1$ 'and $\mathrm{H}-3$ ' showed it having the relative configuration of 22. Further absolute configuration determination was performed by comparing their optical rotation and circular dichroism to those obtained via quantum theory. Other pairs of substrates, D- $(+)-8$ and L-(-)-8, D-(+)-7 and L- $(-)-7$, D- $(+)-6$ and L-(-)-6, were used in the reactions, only one major L-D product formed, there was no D-D, or L-L products in the reactions. In the L-D products, each pair of them was separated into two enantiomers. Their optical rotations for the enantiomers of 23, 24 and $\mathbf{2 5}$ were $+150.3,+127.2$ and +166.7 . The recorded optical rotations for ent-23, ent-24 and ent-25 were $-148.5,-130.2$ and -170.3 , respectively. The results are summarized in Table 1.

Furthermore, as shown in Fig. 3 below, the TLC spots and HPLC retention times of the different products are examined. Clearly the differences among the D-D and D-L products are obvious. For example, the $\mathrm{R}_{\mathrm{f}}$ value of the $\mathrm{D}-\mathrm{D}$ product $4 \mathrm{a}$ from $\mathrm{D}-(+)-\mathbf{1}$ was about 0.8 , while that of $\mathrm{D}-\mathrm{L}$ product derived from $\mathrm{D}-(+)-1$ and $\mathrm{L}-(-)-1$ was about 0.45 . The minor product obtained in the same reaction $\mathbf{4 b}$ had about 0.30 of $R_{f}$ value. When the three compounds were mixed at c point, the three compounds were well separated, and they exhibited the same $R_{f}$ values as the standard point in the left $\left(1^{\#}\right)$. The different $R_{f}$ values between the $\mathrm{D}-\mathrm{D}$ products 13 (derived from $\mathrm{D}-(+)-\mathbf{7}$ $\left(2^{\#}\right)$ ) and D-L products 23 (derived from D-(+)-7 and L-(-)-7)) were also obvious. Similarly, the D-D product 14 from D-(+)$\mathbf{8}$ had different $R_{f}$ value from that of $\mathbf{2 4}$, which was derived from D-(+)-8 and L-(-)-8. The $\mathrm{R}_{\mathrm{f}}$ values of $\mathbf{1 2}$ (D-D product from $\mathrm{D}-(+)-\mathbf{6})$ was the same as that of $\mathbf{2 5}$ which derived from D-(+)-6 and L-(-)-6 in TLC experiments. It looks like that this does not agree with the obtained results. However, they had different retention time (RT) in HPLC using chiral column $\left(4^{\#}\right)$. For example, the D-D product $\mathbf{1 2}$ had about 17.3 minute, L-L product had very close RT of 17.52 minutes. But the D-L product 25 (include its ent-25, L-D product) had 13.7 or 16.1 minute. The experiments confirmed the products formed in the reactions of the $\mathrm{D}$ - and L-amino acid analogue mixtures are different from the use of D- or L-amino acid analogue.
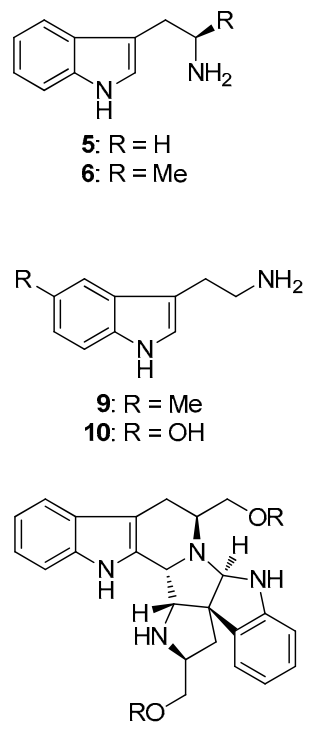

13 (7): $R=A c, 48 \%$ $14(8): R=B z, 52 \%$<smiles>[R]CC(N)Cc1c[nH]c2ccccc12</smiles>

7: $R=A c$

8: $R=B z$

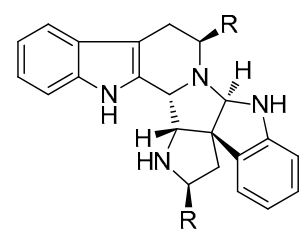

11 (5): $\mathrm{R}=\mathrm{H}, 47 \%$ 12 (6): $\mathrm{R}=\mathrm{Me}, 55 \%$

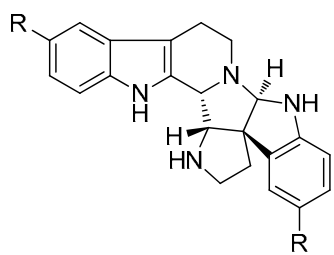

15 (9): $R=M e, 55 \%$ 16 (10): $\mathrm{R}=\mathrm{OH}, 0$

\section{是 Springer}




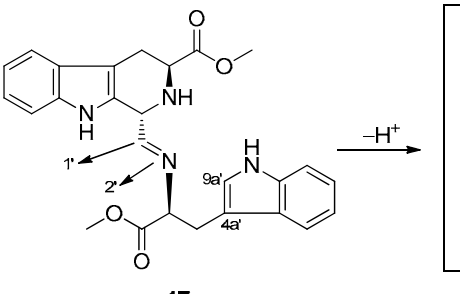

17

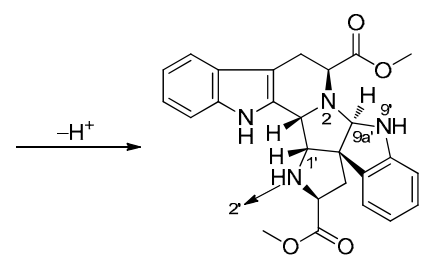

$4 a$

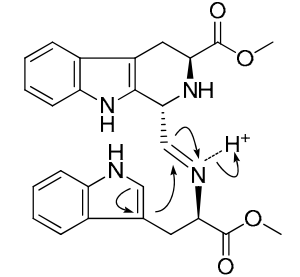

18

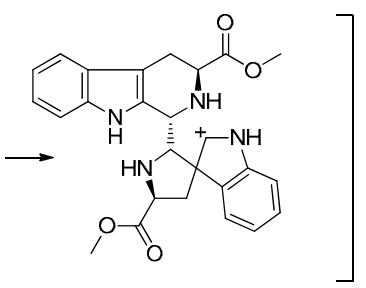

19

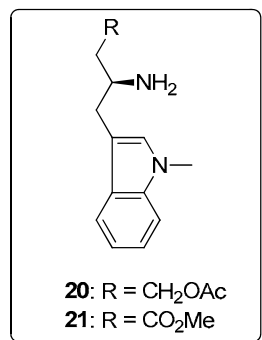

Fig. 2. Proposed mechanisms for formation of $\mathbf{4}$ from intermediate $\mathbf{1 7}$

This special reaction should belong to chirality recognition reaction. However, it may be called as chirality pairing recognition reaction, or molecular sex recognition reaction if D-(+)- or L-(-)-amino acid derivatives are considered as opposite sex pairs. Then their corresponding reaction products as enantiomer mixtures could be expected as natural behavior, which tends to occur in nature. This behavior looks like the sex selectivity of livings.
The discoveries of different chirality selectivities may disclose some secrets during life evolution. For example, if Lamino acids formed with a little more excessive quantity than D-amino acids due to some reasons in oceanic era, the excessive quantity of L-amino acids may be involved in life formation, then the left equal mole of L- and D-amino acids may take similar chirality pairing recognition reactions, because it is easy to form $\mathrm{CHO}-\mathrm{CHO}$ and other simple organic

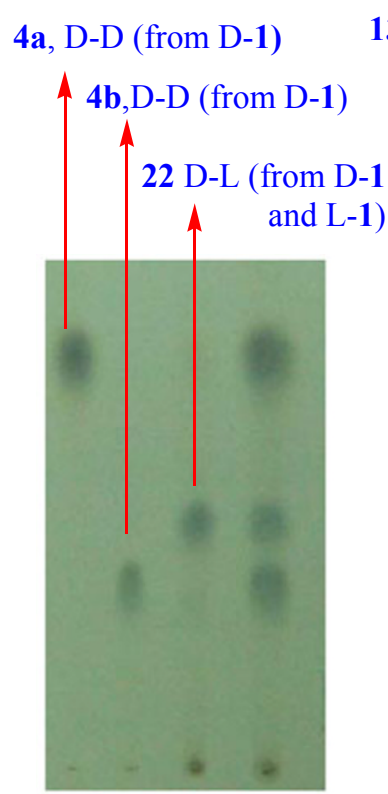

$\begin{array}{llll}\text { a } & \mathbf{a}_{1} & \mathbf{b} & \mathbf{c}\end{array}$

$1 \#$
13, D-D (from D-7)

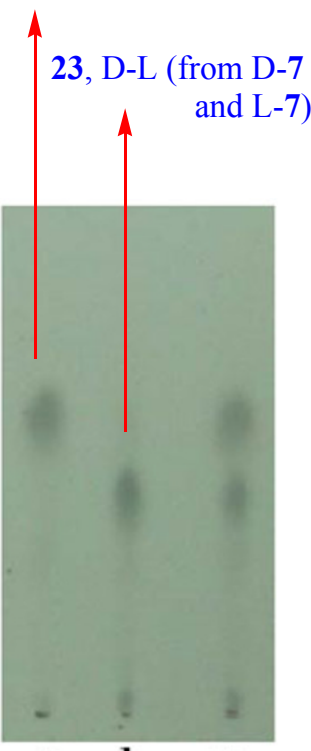

a $\quad$ b $\quad$ c

2\#
14, D-D (from D-8)

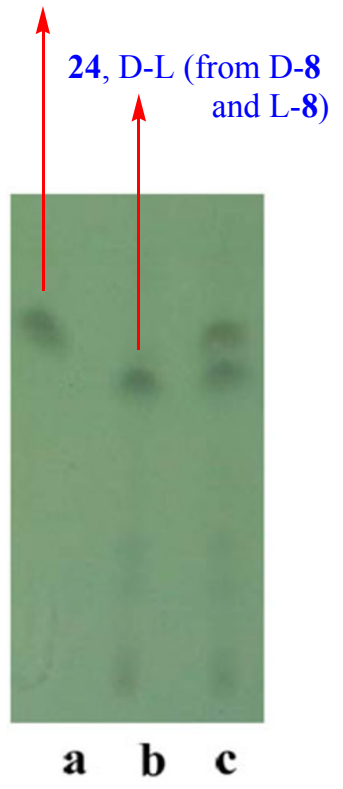

3\#
12, D-D (from D-6)

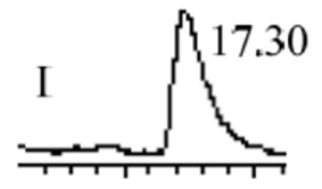

12, L-L (From L-6)

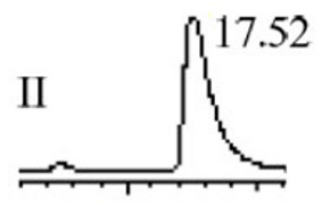

25, D-L (from D-6 and L-6)

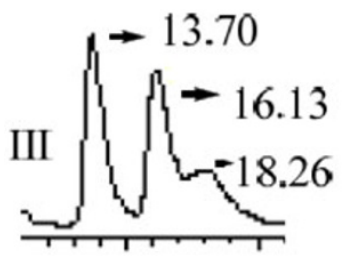

4\#

Fig. 3. TLC analyses $\left(1^{\#}\right.$ to $\left.3^{\#}\right)$, spot a: pure major product (D-D, or L-L) from D- or L-amino acid derivatives; $\mathbf{a}_{1}$ : pure minor product; $\mathbf{b}$ : products in solution of D- and L-amino acid derivatives; $\mathbf{c}$ : mixed $\mathbf{a}$ (and $\mathbf{a}_{1}$ in $2^{\#}$ ) and $\mathbf{b}$ (ethyl acetate:petrol ether $=1: 1$ ). Mixtures of ethyl acetate/petrol ether with $1.5 / 1$ were used in $1^{\#}$ and $1 / 1$ in $2^{\#}$. $\mathrm{CHCl}_{3}$ and methanol $(60: 1)$ was used in $3^{\#}$. In $4^{\#}$, case I is the retention time (RT, minute) of reaction product of D-methyltrypotamine in HPLC using chiral column; case II is the RT for retention time for reaction product of L-methyltrypotamine. Case III is the RT for mixture of D- and L-methyltrypotamine products, and their RTs in HPLC are different from those in cases I and II (their $\mathrm{R}_{\mathrm{f}}$ values in TLC are the same under lab conditions). 
Table 1. Chirality pairing recognition reactions in presence of $\mathrm{CHOCHO}$

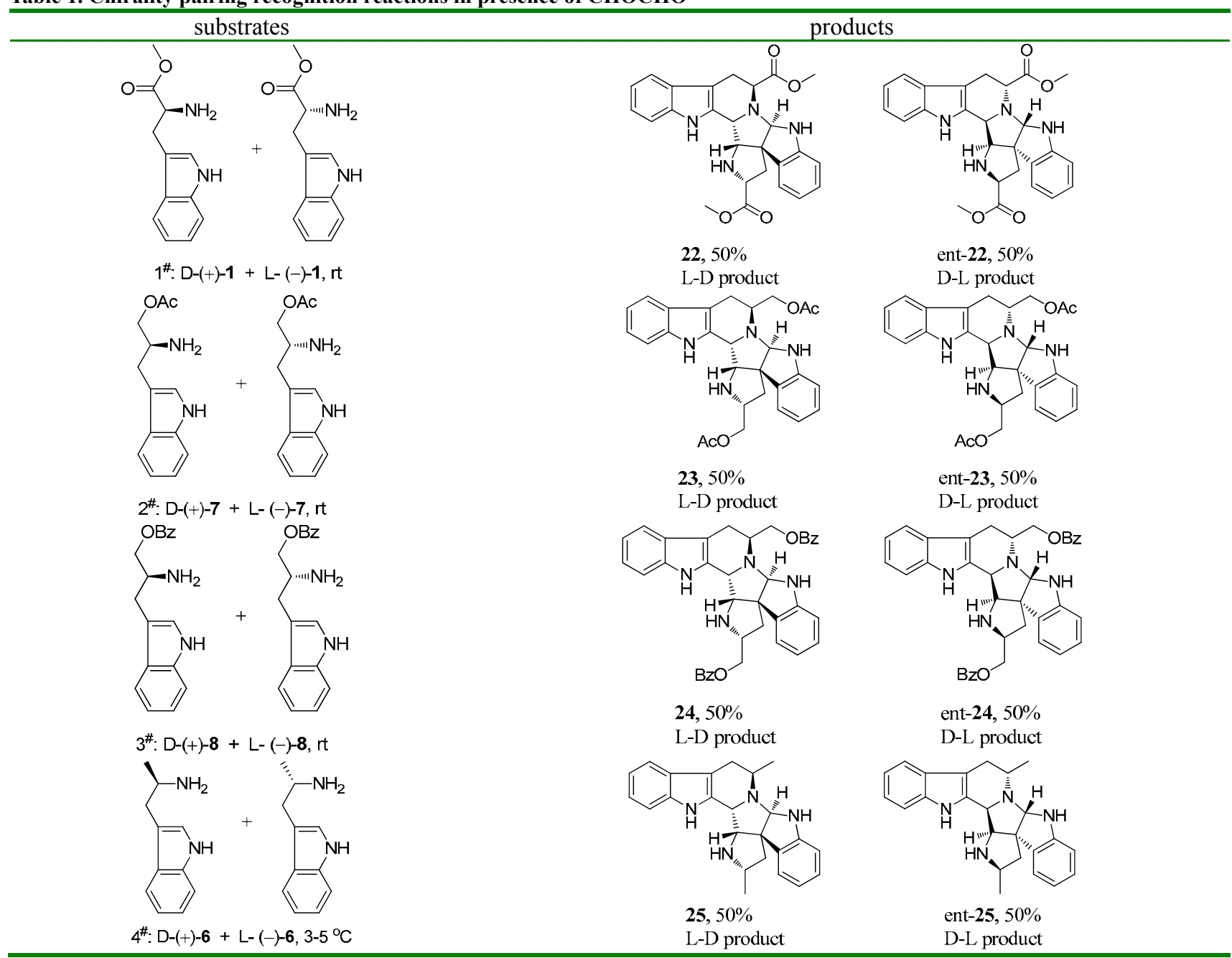

compounds in oceanic era, the tiny acid under neutral conditions can accelerate the reactions. It may be a starting point of a new chemistry: evolution chemistry. Thus, it is possible to construct chirality pairing recognition or molecular sex recognition reaction which can be included in the range of evolution chemistry.

\section{Experimental Section}

General Experimental Procedures. Tryptophan methyl ester solution ( $1.0 \mathrm{mmol}$ in $20 \mathrm{~mL} \mathrm{CH} \mathrm{Cl}_{2}$ ) was cooled using ice-bath. The aldehyde (CHOCHO, $0.6 \mathrm{mmol}$ ) was then injected into the solution. Suitable molecular sieve was added to remove the water formed in Pictet-Spengler reaction. About $20 \mathrm{~h}$ later, 0.01 eq. of TFA was added into the solution for further cycloaddition. This procedure lasted over $40 \mathrm{~h}$. Then the molecular sieve was filtered and the solution was removed under reduced pressure. The mixture was purified using chromatography column by silica gel. Ethyl acetate and petroleum ether mixture was used in the isolation. One major product $4 \mathbf{a}$ was obtained (60\%), and the minor product (23\%) was obtained. Total yield is $83 \%$. Other products can be obtained under the similar procedure when starting materials changed to the corresponding compounds.

4a. ESIMS, $m / z 459[\mathrm{M}+\mathrm{H}]^{+}$. HRMS $m / z$ calcd for $\mathrm{C}_{26} \mathrm{H}_{27} \mathrm{~N}_{4} \mathrm{O}_{4}[\mathrm{M}+\mathrm{H}]^{+} 459.2032$, found 459.2022. $[\alpha]_{\mathrm{D}}^{25}+156.3$ (c $\left.0.16, \mathrm{CHCl}_{3}\right)$. IR (KBr): 3379, 2949, 1736, 1608, 1486, 1466, 1452, 13371, 1199, 1022, $742 \mathrm{~cm}^{-1}$. ${ }^{1} \mathrm{H}$ NMR (500 MHz, $\left.\mathrm{CDCl}_{3}\right) \delta 2.08(1 \mathrm{H}$, br. s, NH-2'), $2.38(1 \mathrm{H}, \mathrm{dd}, J=13.2,9.0$ $\left.\mathrm{Hz}, \mathrm{H}-4^{\prime}\right), 2.50(1 \mathrm{H}, \mathrm{dd}, J=13.2,6.8 \mathrm{~Hz}, \mathrm{H}-4$ '), $3.04(1 \mathrm{H}$, ddd, $J=15.7,3.3,1.6 \mathrm{~Hz}, \mathrm{H}-4), 3.25(1 \mathrm{H}, \mathrm{ddd}, J=15.6,6.7,1.6$ $\mathrm{Hz}, \mathrm{H}-4), 3.64\left(3 \mathrm{H}, \mathrm{s}, \mathrm{COOCH}_{3}-3^{\prime}\right), 3.70\left(3 \mathrm{H}, \mathrm{s}, \mathrm{COOCH}_{3}-3\right)$, $3.90\left(1 \mathrm{H}, \mathrm{dd}, J=9.0,6.8 \mathrm{~Hz}, \mathrm{H}-3{ }^{\prime}\right), 4.10(1 \mathrm{H}, \mathrm{d}, J=5.5 \mathrm{~Hz}$, H-1'), 4.27 (1H, dd, $J=6.6,3.4 \mathrm{~Hz}, \mathrm{H}-3), 4.58(1 \mathrm{H}$, br. s, NH$\left.9^{\prime}\right), 4.63(1 \mathrm{H}, \mathrm{d}, J=5.5 \mathrm{~Hz}, \mathrm{H}-1), 4.97(1 \mathrm{H}, \mathrm{s}, \mathrm{H}-9$ 'a), $6.63(1 \mathrm{H}$, d, $\left.J=7.5 \mathrm{~Hz}, \mathrm{H}-8^{\prime}\right), 6.77\left(1 \mathrm{H}, \mathrm{td}, J=7.5,0.7 \mathrm{~Hz}, \mathrm{H}-6^{\prime}\right), 7.06-$ $7.12(4 \mathrm{H}, \mathrm{m}, \mathrm{H}-5$ ' $, 6,7$ ', 7), $7.29(1 \mathrm{H}, \mathrm{d}, J=6.8 \mathrm{~Hz}, \mathrm{H}-8), 7.46$ $(1 \mathrm{H}, \mathrm{d}, J=7.6 \mathrm{~Hz}, \mathrm{H}-5), 8.32$ (1H, br. s, $\mathrm{NH}-9) .{ }^{13} \mathrm{C}$ NMR $\left(125 \mathrm{MHz}, \mathrm{CDCl}_{3}\right) \delta 24.4(\mathrm{C}-4), 43.3\left(\mathrm{C}-4^{\prime}\right), 52.0\left(\mathrm{OCH}_{3}-3^{\prime}\right)$, $\left.52.2\left(\mathrm{OCH}_{3}-3\right), 56.5(\mathrm{C}-3), 57.1(\mathrm{C}-1), 61.0(\mathrm{C}-3)^{\prime}\right), 64.7(\mathrm{C}-$ 4'a), 72.2 (C-1'), 88.3 (C-9'a), 108.3 (C-4a), 109.5 (C-8'), 111.2 (C-8), 118.0 (C-5), 119.1 (C-6'), 119.4 (C-6), 121.8 (C7), 123.3 (C-7'), 126.8 (C-5a), 128.5 (C-5'), 130.1 (C-5'a), 130.8 (C-9a), 136.5 (C-8a), 150.4 (C-8'a), 174.6 (CO-3'), $174.7(\mathrm{CO}-3)$. 
4b. ESIMS, $m / z 459[\mathrm{M}+\mathrm{H}]^{+}$. HRMS $\mathrm{m} / z$ calcd for $\mathrm{C}_{26} \mathrm{H}_{27} \mathrm{~N}_{4} \mathrm{O}_{4}[\mathrm{M}+\mathrm{H}]^{+}$459.2032, found 459.2046. $[\alpha]_{\mathrm{D}}^{25}-146.7$ (c $\left.0.15, \mathrm{CHCl}_{3}\right)$. IR (KBr): 3382, 2950, 2925, 1737, 1608, 1486, 1467, 1451, 1331, 1260, 1172, 1023, $742 \mathrm{~cm}^{-1}$. ${ }^{1} \mathrm{H}$ NMR $\left(500 \mathrm{MHz}, \mathrm{CDCl}_{3}\right) \delta 2.18\left(1 \mathrm{H}, \mathrm{dd}, J=13.2,7.7 \mathrm{~Hz}, \mathrm{H}-4^{\prime}\right)$, $2.20\left(1 \mathrm{H}\right.$, br. s, NH-2'), $2.64\left(1 \mathrm{H}, \mathrm{dd}, J=13.2,7.5 \mathrm{~Hz}, \mathrm{H}-4^{\prime}\right)$, $3.07(1 \mathrm{H}, \mathrm{dd}, J=13.2,4.6 \mathrm{~Hz}, \mathrm{H}-4), 3.23(1 \mathrm{H}, \mathrm{dd}, J=13.2$, $11.2 \mathrm{~Hz}, \mathrm{H}-4), 3.66\left(3 \mathrm{H}, \mathrm{s}, \mathrm{COOCH}_{3}-3^{\prime}\right), 3.86(3 \mathrm{H}, \mathrm{s}$, $\left.\mathrm{COOCH}_{3}-3\right), 3.92(1 \mathrm{H}, \mathrm{dd}, J=11.2,4.6 \mathrm{~Hz}, \mathrm{H}-3), 4.07(1 \mathrm{H}$, $\left.\mathrm{dd}, J=7.7,7.5 \mathrm{~Hz}, \mathrm{H}-3^{\prime}\right), 4.32(1 \mathrm{H}, \mathrm{d}, J=4.5 \mathrm{~Hz}, \mathrm{H}-1), 4.43$ $\left(1 \mathrm{H}\right.$, br. s, NH-9'), $5.24\left(1 \mathrm{H}, \mathrm{d}, J=4.5,3.0 \mathrm{~Hz}, \mathrm{H}-1^{\prime}\right), 5.24(1 \mathrm{H}$, d, $\left.J=3.0 \mathrm{~Hz}, \mathrm{H}-9^{\prime} \mathrm{a}\right), 6.62\left(1 \mathrm{H}, \mathrm{d}, J=7.5 \mathrm{~Hz}, \mathrm{H}-8^{\prime}\right), 6.79(1 \mathrm{H}$, dd, $J=7.0,6.8 \mathrm{~Hz}, \mathrm{H}-6$ '), $7.06(1 \mathrm{H}, \mathrm{m}, \mathrm{H}-6), 7.07(1 \mathrm{H}, \mathrm{m}, \mathrm{H}-$ 7), 7.08 (1H, m, H-7'), 7.09 (1H, m, H-5'), $7.23(1 \mathrm{H}, \mathrm{d}, J=7.7$ $\mathrm{Hz}, \mathrm{H}-8), 7.47(1 \mathrm{H}, \mathrm{m}, \mathrm{H}-5), 8.85$ (1H, br. s, NH-9). ${ }^{13} \mathrm{C} \mathrm{NMR}$ $\left(125 \mathrm{MHz}, \mathrm{CDCl}_{3}\right) \delta 24.9(\mathrm{C}-4), 43.7\left(\mathrm{C}-4^{\prime}\right), 52.2\left(\mathrm{OCH}_{3}-3^{\prime}\right)$, $52.4\left(\mathrm{OCH}_{3}-3\right), 58.1(\mathrm{C}-3), 59.3(\mathrm{C}-1), 63.1\left(\mathrm{C}-3^{\prime}\right), 63.8\left(\mathrm{C}-4{ }^{\prime} \mathrm{a}\right)$, 72.6 (C-1'), 85.3 (C-9'a), 108.9 (C-4a), 109 (C-8'), 111.4 (C-8), 117.9 (C-5), 119.2 (C-6'), 119.4 (C-6), 121.7 (C-7), 122.7 (C7'), 126.7 (C-5a), 128.5 (C-5'), 130.8 (C-9a), 131.5 (C-5'a), 136.6 (C-8a), 150.4 (C-8'a), 172.5 (CO-3'), 172.8 (CO-3).

11. ESIMS, $m / z 343[\mathrm{M}+\mathrm{H}]^{+}$. HRMS $\mathrm{m} / z$ calcd for $\mathrm{C}_{22} \mathrm{H}_{23} \mathrm{~N}_{4}[\mathrm{M}+\mathrm{H}]^{+}$343.1922, found 343.1930. $[\alpha]_{\mathrm{D}}^{25}+42.6(c$ 0.24, $\mathrm{CHCl}_{3}$ ). IR (KBr): 3382, 2920, 1672, 1609, 1486, 1468, $1337,1178,743 \mathrm{~cm}^{-1}$. ${ }^{1} \mathrm{H}$ NMR $\left(400 \mathrm{MHz}, \mathrm{CDCl}_{3}\right) \delta 2.02-$ $2.08\left(1 \mathrm{H}, \mathrm{m}, \mathrm{H}-4^{\prime} \alpha\right), 2.27-2.34\left(1 \mathrm{H}, \mathrm{m}, \mathrm{H}-4^{\prime} \beta\right), 2.77-2.83(1 \mathrm{H}$, $\mathrm{m}, \mathrm{H}-4 \beta), 2.91-3.01\left(4 \mathrm{H}, \mathrm{m}, \mathrm{H}-3 \alpha, 3^{\prime}, 4 \alpha\right), 3.27-3.33(1 \mathrm{H}, \mathrm{m}$, $\mathrm{H}-3 \beta), 3.86\left(1 \mathrm{H}, \mathrm{d}, J=5.2 \mathrm{~Hz}, \mathrm{H}-1^{\prime}\right), 4.26(1 \mathrm{H}, \mathrm{d}, J=5.1 \mathrm{~Hz}$, H-1), 4.99 (1H, s, H-9'a), 6.56 (1H, d, $\left.J=7.7 \mathrm{~Hz}, \mathrm{H}-8^{\prime}\right), 6.71$ (1H, t, $\left.J=7.3 \mathrm{~Hz}, \mathrm{H}-6^{\prime}\right), 6.99-7.07$ (4H, m, H-6, 7, 5', 7'), 7.29 $(1 \mathrm{H}, \mathrm{d}, J=7.8 \mathrm{~Hz}, \mathrm{H}-8), 7.45(1 \mathrm{H}, \mathrm{d}, J=7.6 \mathrm{~Hz}, \mathrm{H}-5), 9.08$ $(1 \mathrm{H}, \mathrm{s}, \mathrm{N} H-9) .{ }^{13} \mathrm{C} \mathrm{NMR}\left(100 \mathrm{MHz}, \mathrm{CDCl}_{3}\right) \delta 21.1,41.3,43.5$, 49.0, 58.0, 64.0, 72.9, 87.6, 109.4, 110.2, 111.4, 117.9, 119.2, $119.3,121.5,122.9,127.1,128.2,131.7,132.0,136.5,150.5$.

12. ESIMS, $m / z 371[\mathrm{M}+\mathrm{H}]^{+}$. HRMS $m / z$ calcd for $\mathrm{C}_{24} \mathrm{H}_{27} \mathrm{~N}_{4}[\mathrm{M}+\mathrm{H}]^{+} 371.2235$, found 371.2233. $[\alpha]_{\mathrm{D}}^{25}-269.8(c$ $0.22, \mathrm{CHCl}_{3}$ ). IR (KBr): $3428,2918,1608,1470,1336,1188$, $740 \mathrm{~cm}^{-1} .{ }^{1} \mathrm{H}$ NMR $\left(500 \mathrm{MHz}, \mathrm{CDCl}_{3}\right) \delta 1.21(3 \mathrm{H}, \mathrm{d}, J=6.2$ $\left.\mathrm{Hz}, \mathrm{CH}_{3}-3\right), 1.44\left(3 \mathrm{H}, \mathrm{d}, J=6.2 \mathrm{~Hz}, \mathrm{CH}_{3}-3^{\prime}\right), 1.62(1 \mathrm{H}, \mathrm{dd}, J=$ $\left.13.2,11.2 \mathrm{~Hz}, \mathrm{H}-4^{\prime} \alpha\right), 2.59-2.65\left(2 \mathrm{H}, \mathrm{m}, \mathrm{H}-4 \alpha, 4^{\prime} \beta\right), 2.90(1 \mathrm{H}$, ddd, $J=14.9,4.0,1.3 \mathrm{~Hz}, \mathrm{H}-4 \beta), 3.20-3.24(1 \mathrm{H}, \mathrm{m}, \mathrm{H}-3)$, $3.42-3.47\left(1 \mathrm{H}, \mathrm{m}, \mathrm{H}-3^{\prime}\right), 3.83\left(1 \mathrm{H}, \mathrm{d}, J=5.1 \mathrm{~Hz}, \mathrm{H}-1^{\prime}\right), 4.00$ $\left(1 \mathrm{H}, \mathrm{d}, J=3.4 \mathrm{~Hz}, \mathrm{~N} H-9^{\prime}\right), 4.30(1 \mathrm{H}, \mathrm{d}, J=5.0 \mathrm{~Hz}, \mathrm{H}-1), 5.24$ (1H, d, $J=3.4 \mathrm{~Hz}, \mathrm{H}-9$ 'a), $6.65\left(1 \mathrm{H}, \mathrm{d}, J=7.7 \mathrm{~Hz}, \mathrm{H}-8^{\prime}\right), 6.84$ $\left(1 \mathrm{H}, \mathrm{t}, J=7.3 \mathrm{~Hz}, \mathrm{H}^{-6} \mathbf{6}^{\prime}\right), 7.05-7.12\left(4 \mathrm{H}, \mathrm{m}, \mathrm{H}-6,7,5^{\prime}, 7^{\prime}\right), 7.20$ $(1 \mathrm{H}, \mathrm{m}, \mathrm{H}-8), 7.44(1 \mathrm{H}, \mathrm{m}, \mathrm{H}-5), 9.10\left(1 \mathrm{H}\right.$, br. s, NH-9). ${ }^{13} \mathrm{C}$ NMR $\left(100 \mathrm{MHz}, \mathrm{CDCl}_{3}\right) \delta 18.9,20.5,31.2,49.8,51.2,57.6$, 58.4, 64.1, 75.2, 86.7, 109.1, 110.2, 111.4, 117.7, 119.1, 119.2, $121.4,122.7,126.7,128.1,131.2,132.6,136.5,150.4$.

13. ESIMS, $m / z 487[\mathrm{M}+\mathrm{H}]^{+}$. HRMS $m / z$ calcd for $\mathrm{C}_{28} \mathrm{H}_{31} \mathrm{~N}_{4} \mathrm{O}_{4}[\mathrm{M}+\mathrm{H}]^{+}$487.2345, found 487.2339. $[\alpha]_{\mathrm{D}}^{25}+115.2$ (c $0.83, \mathrm{CHCl}_{3}$ ). IR (KBr): 3394, 2924, 1736, 1608, 1465, $1237,1035,741 \mathrm{~cm}^{-1} .{ }^{1} \mathrm{H}$ NMR $\left(500 \mathrm{MHz}, \mathrm{CDCl}_{3}\right) \delta 1.91(1 \mathrm{H}$, dd, $\left.J=12.8,10.7 \mathrm{~Hz}, \mathrm{H}-4^{\prime} \alpha\right), 2.03\left(3 \mathrm{H}, \mathrm{s}, \mathrm{H}-\mathrm{OCH}_{3}\right), 2.04(3 \mathrm{H}$, s, $\left.\mathrm{H}-\mathrm{OCH}_{3}\right), 2.20\left(1 \mathrm{H}, \mathrm{dd}, J=15.7,5.5 \mathrm{~Hz}, \mathrm{H}-4^{\prime} \beta\right), 2.87(1 \mathrm{H}$, d, $J=15.3 \mathrm{~Hz}, \mathrm{H}-4 \beta), 3.05(1 \mathrm{H}, \mathrm{dd}, J=15.2,7.1 \mathrm{~Hz}, \mathrm{H}-4 \alpha)$, $3.221 \mathrm{H},\left(\mathrm{m}, \mathrm{H}-3^{\prime}\right), 3.72(1 \mathrm{H}, \mathrm{m}, \mathrm{H}-3), 3.84(1 \mathrm{H}, \mathrm{dd}, J=13.0$, $\left.10.8 \mathrm{~Hz}, \mathrm{H}-3-\mathrm{CH}_{2}(\alpha) \mathrm{OAc}\right), 3.96(1 \mathrm{H}, \mathrm{dd}, J=15.2,6.5 \mathrm{~Hz}, \mathrm{H}-$ $\left.3^{\prime}-\mathrm{CH}_{2}(\alpha) \mathrm{OAc}\right), 4.04\left(2 \mathrm{H}, \mathrm{m}, \mathrm{H}-1^{\prime}, 3^{\prime}-\mathrm{CH}_{2}(\beta) \mathrm{OAc}\right), 4.45(1 \mathrm{H}$, $\mathrm{d}, J=5.3 \mathrm{~Hz}, \mathrm{H}-1), 4.60(1 \mathrm{H}, \mathrm{dd}, J=10.6,4.4 \mathrm{~Hz}, \mathrm{H}-3-$ $\left.\mathrm{CH}_{2}(\beta) \mathrm{OAc}\right), 4.95(1 \mathrm{H}, \mathrm{s}, \mathrm{H}-9 ' \mathrm{a}), 6.64(1 \mathrm{H}, \mathrm{d}, J=7.7 \mathrm{~Hz}, \mathrm{H}-$ $\left.8^{\prime}\right), 6.80(1 \mathrm{H}, \mathrm{t}, J=7.8 \mathrm{~Hz}, \mathrm{H}-6$ '), 7.08-7.20 (4H, m, H-6, 7, 5', 7 '), $7.31(1 \mathrm{H}, \mathrm{d}, J=8.0 \mathrm{~Hz}, \mathrm{H}-8), 7.52(1 \mathrm{H}, \mathrm{d}, J=7.8 \mathrm{~Hz}, \mathrm{H}-$ 5), $8.47(1 \mathrm{H}, \mathrm{s}, \mathrm{N} H-9) .{ }^{13} \mathrm{C}$ NMR $\left(100 \mathrm{MHz}^{-} \mathrm{CDCl}_{3}\right) \delta 20.8$, $20.9,22.3,42.4,51.3,55.5,58.2,64.2,64.5,66.7,71.6,86.3$, $108.8,109.6,111.1,118.0,119.1,119.4,121.9,122.9,127.5$, $128.3,130.5,131.6,136.7,150.3,170.9,171.3$.

14. ESIMS, $m / z 611[\mathrm{M}+\mathrm{H}]^{+}$. HRMS $\mathrm{m} / z$ calcd for $\mathrm{C}_{38} \mathrm{H}_{35} \mathrm{~N}_{4} \mathrm{O}_{4}[\mathrm{M}+\mathrm{H}]^{+}$611.2658, found 611.2653. $[\alpha]_{\mathrm{D}}^{25}-166.7$ (c $0.3, \mathrm{CHCl}_{3}$ ). IR (KBr): 3384, 2919, 1719, 1605, 1466, 1272, $1112,747,710 \mathrm{~cm}^{-1} .{ }^{1} \mathrm{H}$ NMR $\left(500 \mathrm{MHz}, \mathrm{CDCl}_{3}\right) \delta 1.93(1 \mathrm{H}$, dd, $\left.J=13.3,10.5 \mathrm{~Hz}, \mathrm{H}-4^{\prime} \alpha\right), 2.55(1 \mathrm{H}, \mathrm{dd}, J=13.3,6.3 \mathrm{~Hz}$, $\left.\mathrm{H}-4^{\prime} \beta\right), 2.80(1 \mathrm{H}, \mathrm{dd}, J=12.9,8.7 \mathrm{~Hz}, \mathrm{H}-4 \beta), 3.00(1 \mathrm{H}, \mathrm{dd}, J$ $=15.1,3.6 \mathrm{~Hz}, \mathrm{H}-4 \alpha), 3.57(1 \mathrm{H}, \mathrm{m}, \mathrm{H}-3 \mathrm{\prime}), 3.71(1 \mathrm{H}, \mathrm{m}, \mathrm{H}-3)$, $3.78\left(1 \mathrm{H}, \mathrm{d}, J=5.0 \mathrm{~Hz}, \mathrm{H}-1^{\prime}\right), 4.31-4.38(3 \mathrm{H}, \mathrm{m}, \mathrm{H}-3-$ $\mathrm{CH}_{2}$ (a)Obz, H-3'- $\left.\mathrm{CH}_{2} \mathrm{OBz}\right), 4.69$ (2H, m, H-1, H-3$\left.\mathrm{CH}_{2}(\mathrm{~b}) \mathrm{OBz}\right), 5.33(1 \mathrm{H}, \mathrm{s}, \mathrm{H}-9$ 'a), $6.47(1 \mathrm{H}, \mathrm{d}, J=7.7 \mathrm{~Hz}, \mathrm{H}-$ 8'), 6.79 (1H, t, $J=7.4 \mathrm{~Hz}, \mathrm{H}-6$ '), 7.03-7.07 (4H, m, H-6, 7, 5', 7 '), $7.13(1 \mathrm{H}, \mathrm{d}, J=7.4 \mathrm{~Hz}, \mathrm{H}-8), 7.37(2 \mathrm{H}, \mathrm{t}, J=7.7 \mathrm{~Hz}, \mathrm{H}-$ Bz), 7.49-7.63 (5H, m, H-5, H-Bz), $7.90(2 \mathrm{H}, \mathrm{d}, J=7.3 \mathrm{~Hz}$, $\mathrm{H}-\mathrm{Bz}), 8.08(2 \mathrm{H}, \mathrm{d}, J=7.3 \mathrm{~Hz}, \mathrm{H}-\mathrm{Bz}), 8.72(1 \mathrm{H}, \mathrm{s}, N \mathrm{H}-9) .{ }^{13} \mathrm{C}$ NMR $\left(100 \mathrm{MHz}, \mathrm{CDCl}_{3}\right) \delta 25.9,45.2,53.5,58.2,61.2,64.2$, $64.9,67.9,74.5,87.1,108.9,109.0,111.2,117.8,119.1,119.3$, $121.6,122.7,126.8,128.2,128.3,128.8,129.4,129.5,129.6$, $129.8,131.2,132.5,133.1,133.5,136.6,150.3,166.1,166.2$.

15. ESIMS, $m / z 371[\mathrm{M}+\mathrm{H}]^{+}$. HRMS $m / z$ calcd for $\mathrm{C}_{24} \mathrm{H}_{27} \mathrm{~N}_{4}[\mathrm{M}+\mathrm{H}]^{+}$371.2235, found 371.2234. $[\alpha]_{\mathrm{D}}^{25}+120(c$ $0.13, \mathrm{CHCl}_{3}$ ). IR (KBr): 3387, 2914, 1661, 1621, 1498, 1202, $1142,803,732 \mathrm{~cm}^{-1} .{ }^{1} \mathrm{H}$ NMR $\left(500 \mathrm{MHz}, \mathrm{CDCl}_{3}\right) \delta 2.18(1 \mathrm{H}$, $\left.\mathrm{m}, \mathrm{H}-4^{\prime} \alpha\right), 2.28\left(3 \mathrm{H}, \mathrm{s}, \mathrm{CH}_{3}\right), 2.40\left(3 \mathrm{H}, \mathrm{s}, \mathrm{CH}_{3}\right), 2.45(1 \mathrm{H}, \mathrm{m}$, $\mathrm{H}-4 \beta), 2.74(2 \mathrm{H}, \mathrm{m}, \mathrm{H}-4), 2.92-3.23\left(4 \mathrm{H}, \mathrm{m}, \mathrm{H}-3,3^{\prime}\right), 4.04$ $\left(1 \mathrm{H}\right.$, br. s, $\left.N \mathrm{H}-9^{\prime}\right), 4.18\left(1 \mathrm{H}, \mathrm{s}, \mathrm{H}-1^{\prime}\right), 4.22(1 \mathrm{H}, \mathrm{s}, \mathrm{H}-1), 5.05$ $\left(1 \mathrm{H}, \mathrm{s}, \mathrm{H}-9{ }^{\prime} \mathrm{a}\right), 6.51\left(1 \mathrm{H}, \mathrm{d}, J=8.7 \mathrm{~Hz}, \mathrm{H}-8^{\prime}\right), 6.93-7.18(5 \mathrm{H}$, m, H-Ar), $9.86(1 \mathrm{H}, \mathrm{s}, N \mathrm{H}-9) .{ }^{13} \mathrm{C}$ NMR $\left(100 \mathrm{MHz} \mathrm{CDCl}_{3}\right) \delta$ $20.9,21.6,21.8,37.5,43.3,47.1,56.5,63.2,70.5,88.2,109.8$, $111.3,111.6,117.9,123.8,124.1,127.1,127.4,128.6,128.8$, $129.5,130.1,135.3,148.7$.

22. Racemic mixture was resolved by HPLC on a Chiralcell-OD-H column $(250 \times 10 \mathrm{~mm}, n$-hexane $/ i$-PrOH $=85 / 15)$. $[\alpha]_{\mathrm{D}}^{25}+187.3\left(c 0.12, \mathrm{CHCl}_{3}\right)$. ESIMS, $m / z 459[\mathrm{M}+\mathrm{H}]^{+}$. HRMS $m / z$ calcd for $\mathrm{C}_{26} \mathrm{H}_{27} \mathrm{~N}_{4} \mathrm{O}_{4},[\mathrm{M}+\mathrm{H}]^{+} 459.2032$, found 459.2026. ${ }^{1} \mathrm{H}$ NMR $\left(500 \mathrm{MHz}, \mathrm{CDCl}_{3}\right) \delta 2.28(1 \mathrm{H}, \mathrm{dd}, J=$ 13.5, 7.7 Hz, H-4' $\alpha), 2.68\left(1 \mathrm{H}, \mathrm{dd}, J=13.5,7.7 \mathrm{~Hz}, \mathrm{H}^{\prime} 4^{\prime} \beta\right)$, $3.07(1 \mathrm{H}, \mathrm{d}, J=15.7 \mathrm{~Hz}, \mathrm{H}-4 \beta), 3.32(1 \mathrm{H}, \mathrm{dd}, J=15.6,7.1 \mathrm{~Hz}$, $\mathrm{H}-4 \alpha), 3.64\left(3 \mathrm{H}, \mathrm{s}, \mathrm{COOCH}_{3}-3\right), 3.66\left(3 \mathrm{H}, \mathrm{s}, \mathrm{COOCH}_{3}-3^{\prime}\right)$, $3.84\left(1 \mathrm{H}, \mathrm{d}, J=4.5 \mathrm{~Hz}, \mathrm{H}-1^{\prime}\right), 4.00\left(1 \mathrm{H}, \mathrm{t}, J=7.8 \mathrm{~Hz}, \mathrm{H}-3^{\prime}\right)$, $4.28(1 \mathrm{H}, \mathrm{d}, J=6.7 \mathrm{~Hz}, \mathrm{H}-3), 4.50(1 \mathrm{H}$, br. s, NH-9'), 4.70 $(1 \mathrm{H}, \mathrm{d}, J=3.7 \mathrm{~Hz}, \mathrm{H}-1), 5.06(1 \mathrm{H}, \mathrm{s}, \mathrm{H}-9$ 'a $), 6.60(1 \mathrm{H}, \mathrm{d}, J=$ $\left.7.8 \mathrm{~Hz}, \mathrm{H}-8^{\prime}\right), 6.80\left(1 \mathrm{H}, \mathrm{t}, J=7.4 \mathrm{~Hz}, \mathrm{H}-6^{\prime}\right), 7.08-7.12(4 \mathrm{H}, \mathrm{m}$, H-6, 7, 5', 7'), $7.21(1 \mathrm{H}, \mathrm{d}, J=7.9 \mathrm{~Hz}, \mathrm{H}-8), 7.48(1 \mathrm{H}, \mathrm{d}, J=$ $7.0 \mathrm{~Hz}, \mathrm{H}-5), 8.48(1 \mathrm{H}, \mathrm{s}, \mathrm{N} H-9) .{ }^{13} \mathrm{C}$ NMR $\left(125 \mathrm{MHz}, \mathrm{CDCl}_{3}\right)$ $\delta 24.5,44.3,51.9,52.2,55.2,56.2,62.4,64.4,74.1,88.2$, $108.3,109.5,111.2,117.9,119.1,119.3,121.7,122.8,126.9$, $128.5,130.4,131.0,136.6,150.7,173.0,174.8$. The ent-22 
had the -189.2 of optical rotation values in chloroform.

23. Racemic mixture was resolved by HPLC on a Chiralcell-OD-H column $(250 \times 10 \mathrm{~mm}, n$-hexane $/ i$-PrOH $=$ 87/17). $[\alpha]_{\mathrm{D}}^{25}+150.3\left(c 0.45, \mathrm{CHCl}_{3}\right)$. ESIMS, $m / z 487[\mathrm{M}+$ $\mathrm{H}]^{+}$. HRMS $m / z$ calcd for $\mathrm{C}_{28} \mathrm{H}_{31} \mathrm{~N}_{4} \mathrm{O}_{4},[\mathrm{M}+\mathrm{H}]^{+} 487.2345$, found 487.2364. ${ }^{1} \mathrm{H} \mathrm{NMR}\left(500 \mathrm{MHz}, \mathrm{CDCl}_{3}\right) \delta 1.62(1 \mathrm{H}, \mathrm{dd}, J$ $\left.=12.9,10.6 \mathrm{~Hz}, \mathrm{H}-4^{\prime} \alpha\right), 1.95\left(3 \mathrm{H}, \mathrm{s}, \mathrm{OCOCH}_{3}-3^{\prime}\right), 2.07(3 \mathrm{H}, \mathrm{s}$, $\left.\mathrm{OCOCH}_{3}-3\right), 2.35\left(1 \mathrm{H}, \mathrm{dd}, J=16.5,8.0 \mathrm{~Hz}, \mathrm{H}-4^{\prime} \beta\right), 2.90(1 \mathrm{H}$, $\mathrm{d}, J=15.6 \mathrm{~Hz}, \mathrm{H}-4 \beta), 3.08(1 \mathrm{H}, \mathrm{dd}, J=15.7,7.5 \mathrm{~Hz}, \mathrm{H}-4 \alpha)$, $3.73\left(2 \mathrm{H}, \mathrm{m}, \mathrm{H}-3,3^{\prime}\right), 3.88$ (2H, m, H-1', $\left.\mathrm{CH}_{2}(\alpha) \mathrm{OAc}-3\right), 4.03$ $\left(2 \mathrm{H}, \mathrm{m}, \mathrm{CH}_{2} \mathrm{OAc}-3^{\prime}\right), 4.45(1 \mathrm{H}, \mathrm{d}, J=5.2 \mathrm{~Hz}, \mathrm{H}-1), 4.52(1 \mathrm{H}$, br. s, $\left.\mathrm{N} H-9^{\prime}\right), 4.72\left(1 \mathrm{H}, \mathrm{dd}, J=10.7,3.7 \mathrm{~Hz}, \mathrm{CH}_{2}(\beta) \mathrm{OAc}-3\right)$, $5.09\left(1 \mathrm{H}, \mathrm{s}, \mathrm{H}-9\right.$ 'a), $6.63\left(1 \mathrm{H}, \mathrm{d}, J=7.8 \mathrm{~Hz}, \mathrm{H}-8^{\prime}\right), 6.78(1 \mathrm{H}, \mathrm{t}$, $\left.J=7.4 \mathrm{~Hz}, \mathrm{H}-6^{\prime}\right), 7.06-7.12\left(4 \mathrm{H}, \mathrm{m}, \mathrm{H}-6,7,5^{\prime}, 7^{\prime}\right), 7.24(1 \mathrm{H}, \mathrm{d}$, $J=6.7 \mathrm{~Hz}, \mathrm{H}-8), 7.51(1 \mathrm{H}, \mathrm{d}, J=6.7 \mathrm{~Hz}, \mathrm{H}-5), 8.78(1 \mathrm{H}, \mathrm{s}$, $\mathrm{NH}-9) .{ }^{13} \mathrm{C}$ NMR $\left(125 \mathrm{MHz}, \mathrm{CDCl}_{3}\right) \delta 20.8,21.0,23.3,44.4$, $52.0,53.8,60.8,63.7,64.4,66.0,74.4,88.0,108.2,109.4$, $111.3,117.9,119.1,119.2,121.7,122.7,127.4,128.2,130.6$, $132.9,136.6,150.1,170.9,171.3$. The ent-23 had the -148.5 of optical rotation values in chloroform.

24. Racemic mixture was resolved by HPLC on a Chiralcell-OD-H column $(250 \times 10 \mathrm{~mm}, n$-hexane $/ i$-PrOH $=85 / 15)$. $[\alpha]_{\mathrm{D}}^{25}+127.6\left(c\right.$ 0.47, $\left.\mathrm{CHCl}_{3}\right)$. ESIMS, $m / z 611[\mathrm{M}+\mathrm{H}]^{+}$. HRMS $m / z$ calcd for $\mathrm{C}_{38} \mathrm{H}_{35} \mathrm{~N}_{4} \mathrm{O}_{4},[\mathrm{M}+\mathrm{H}]^{+} 611.2658$, found 611.2650. ${ }^{1} \mathrm{H}$ NMR $\left(500 \mathrm{MHz}, \mathrm{CDCl}_{3}\right) \delta 1.84(1 \mathrm{H}, \mathrm{dd}, J=$ $\left.12.9,10.5 \mathrm{~Hz}, \mathrm{H}-4^{\prime} \alpha\right), 2.44\left(1 \mathrm{H}, \mathrm{dd}, J=13.1,6.3 \mathrm{~Hz}, \mathrm{H}-4{ }^{\prime} \beta\right)$, $3.02(1 \mathrm{H}, \mathrm{d}, J=15.5 \mathrm{~Hz}, \mathrm{H}-4 \beta), 3.16(1 \mathrm{H}, \mathrm{dd}, J=16.1,7.0 \mathrm{~Hz}$, $\mathrm{H}-4 \alpha), 3.84\left(1 \mathrm{H}, \mathrm{m}, \mathrm{H}-3^{\prime}\right), 3.92\left(2 \mathrm{H}, \mathrm{m}, \mathrm{H}-3,1^{\prime}\right), 4.14(1 \mathrm{H}, \mathrm{dd}$, $\left.J=10.5,8.7 \mathrm{~Hz}, \mathrm{CH}_{2}(\mathrm{a}) \mathrm{OBz}-3\right), 4.27(1 \mathrm{H}, \mathrm{dd}, J=14.5,3.9 \mathrm{~Hz}$, $\mathrm{CH}_{2}$ (a)OBz-3'), $4.44\left(1 \mathrm{H}, \mathrm{dd}, J=14.5,7.0 \mathrm{~Hz}, \mathrm{CH}_{2}(\mathrm{~b}) \mathrm{OBz}-3^{\prime}\right)$, $4.54(1 \mathrm{H}, \mathrm{d}, \mathrm{H}-1), 4.58(1 \mathrm{H}$, br. s, NH-9'), $5.01(1 \mathrm{H}, \mathrm{dd}, J=$ 13.5, $\left.4.5 \mathrm{~Hz}, \mathrm{CH}_{2}(\mathrm{~b}) \mathrm{OBz}-3\right), 5.20(1 \mathrm{H}, \mathrm{s}, \mathrm{H}-9 \mathrm{\prime}), 6.58(1 \mathrm{H}, \mathrm{d}$, $\left.J=7.8 \mathrm{~Hz}, \mathrm{H}-8^{\prime}\right), 6.79\left(1 \mathrm{H}, \mathrm{t}, J=8.7 \mathrm{~Hz}, \mathrm{H}-6^{\prime}\right), 7.07-7.15(4 \mathrm{H}$, $\left.\mathrm{m}, \mathrm{H}-6,7,5^{\prime}, 7^{\prime}\right), 7.28(1 \mathrm{H}, \mathrm{m}, \mathrm{H}-8), 7.39(2 \mathrm{H}, \mathrm{t}, J=7.7 \mathrm{~Hz}$, H-Bz), 7.44 (2H, t, $J=7.7 \mathrm{~Hz}, \mathrm{H}-\mathrm{Bz}), 7.51-7.59$ (3H, m, H-5, $\mathrm{H}-\mathrm{Bz}), 7.93(2 \mathrm{H}, \mathrm{d}, J=7.5 \mathrm{~Hz}, \mathrm{H}-\mathrm{Bz}), 7.98(2 \mathrm{H}, \mathrm{d}, J=7.5 \mathrm{~Hz}$, $\mathrm{H}-\mathrm{Bz}), 8.86(1 \mathrm{H}, \mathrm{s}, \mathrm{NH}-9) .{ }^{13} \mathrm{C}$ NMR $\left(125 \mathrm{MHz} \mathrm{CDCl}_{3}\right) \delta$ 23.7, 44.5, 52.2, 53.9, 60.9, 64.0, 65.0, 65.6, 74.5, 88.4, 108.4, $109.5,111.3,117.9,119.1,119.2,121.8,122.7,127.4,128.2$, $128.3,128.4,129.5,129.6,129.8,130.1,130.6,130.9,132.8$, $133.1,136.7,150.2,166.2,166.8$. The ent-24 had the -130.2 of optical rotation values in chloroform.

25. Racemic mixture was separated by HPLC on a Chiralcell-OD-H column $(250 \times 10 \mathrm{~mm}, n$-hexane $/ i$ - $\mathrm{PrOH}=92 / 8)$. $[\alpha]_{\mathrm{D}}^{25}+166.7\left(c \quad 0.12, \mathrm{CHCl}_{3}\right.$ ). ESIMS, $m / z 371[\mathrm{M}+\mathrm{H}]^{+}$. HRMS $m / z$ calcd for $\mathrm{C}_{24} \mathrm{H}_{27} \mathrm{~N}_{4},[\mathrm{M}+\mathrm{H}]^{+} 371.2235$, found 371.2238. ${ }^{1} \mathrm{H}$ NMR (600 MHz, $\left.\mathrm{CDCl}_{3}\right) \delta 1.13(3 \mathrm{H}, \mathrm{d}, J=6.3$
$\left.\mathrm{Hz}, \mathrm{CH}_{3}-3\right), 1.25\left(3 \mathrm{H}, \mathrm{d}, J=6.6 \mathrm{~Hz}, \mathrm{CH}_{3}-3^{\prime}\right), 1.43(1 \mathrm{H}, \mathrm{dd}, J=$ $\left.12.6,10.8 \mathrm{~Hz}, \mathrm{H}-4^{\prime} \alpha\right), 2.44\left(1 \mathrm{H}, \mathrm{dd}, J=13.6,5.6 \mathrm{~Hz}, \mathrm{H}-4^{\prime} \beta\right)$, $2.59(1 \mathrm{H}, \mathrm{d}, J=15.5 \mathrm{~Hz}, \mathrm{H}-4 \alpha$, ), $3.16(1 \mathrm{H}, \mathrm{dd}, J=15.0,5.9$ $\mathrm{Hz}, \mathrm{H}-4 \beta), 3.46(1 \mathrm{H}, \mathrm{m}, \mathrm{H}-3 \mathrm{\prime}), 3.76(1 \mathrm{H}, \mathrm{m}, \mathrm{H}-3), 3.82(1 \mathrm{H}, \mathrm{d}$, $\left.J=5.7 \mathrm{~Hz}, \mathrm{H}-1^{\prime}\right), 4.07(1 \mathrm{H}$, br. s, NH-9'), $4.43(1 \mathrm{H}, \mathrm{d}, J=5.6$ $\mathrm{Hz}, \mathrm{H}-1), 5.07$ (1H, s, H-9'a), 6.59 (1H, d, $\left.J=7.7 \mathrm{~Hz}, \mathrm{H}-8^{\prime}\right)$, $6.76\left(1 \mathrm{H}, \mathrm{t}, J=7.5 \mathrm{~Hz}, \mathrm{H}-6^{\prime}\right), 7.04-7.11\left(4 \mathrm{H}, \mathrm{m}, \mathrm{H}-6,7,5^{\prime}, 7^{\prime}\right)$, $7.18(1 \mathrm{H}, \mathrm{dd}, J=6.1,2.8 \mathrm{~Hz}, \mathrm{H}-8), 7.48(1 \mathrm{H}, \mathrm{dd}, J=6.4,2.6$ $\mathrm{Hz}, \mathrm{H}-5), 8.93$ (1H, br. s, NH-9). ${ }^{13} \mathrm{C}$ NMR $\left(150 \mathrm{MHz} \mathrm{CDCl}_{3}\right)$ $\delta 16.8,19.6,28.7,48.5,51.3,52.5,58.3,64.7,74.9,88.9$, $108.9,109.1,111.2,117.8,118.9,119.0,121.4,122.7,127.7$, $127.9,130.9,133.5,136.6,150.3$. The ent-25 had the -170.3 of optical rotation values in chloroform..

\section{Electronic Supplementary Material}

Supplementary material is available in the online version of this article at http://dx.doi.org/10.1007/s13659-012-0003-6 and is accessible for authorized users.

\section{Acknowledgments}

Hua-Jie Zhu thanks the financial supports from NSFC (30873141), 973 Program (2009CB522300), Hebei University and the State Key Laboratory of Phytochemistry and Plant Resources in West China.

Open Access This article is distributed under the terms of the Creative Commons Attribution License which permits any use, distribution, and reproduction in any medium, provided the original author(s) and source are credited.

\section{References}

[1] (a) Rich, A. J. Biol. Chem. 2006, 281, 7693-7696. (b) Forsdyke, D. R. J. Theo. Biology. 2007, 249, 325-330.

[2] Ikai, T.; Okamoto, Y. Chem. Rev. 2009, 109, 6077-6101.

[3] Bai, B.; Shen, L.; Ren, J.; Zhu, H. J. Adv. Syn. \& Cat. 2011, accepted. Doi:10.1002/adsc.201100592.

[4] Bou-Hamdan, F. R.; Leighton, J. L. Angew. Chem. Int. Ed. 2009, 48, 2403-2406.

[5] (a) Intermediate $\mathbf{2}$ reacted with $\mathbf{1}$ formed only $(E)$-imines since the calculated energy of formation of the $(Z)$-imine is $5.1 \mathrm{kcal} / \mathrm{mol}$ higher than that for the $(E)$-imine in $\mathrm{CH}_{2} \mathrm{Cl}_{2}$. Two products were isolated in ratios of $2: 1$ to $3: 1$ after subsequent reactions with trans-2 and cis-2 (theoretically 4:1 at the B3LYP/6-31G(d) level), respectively. (b) Nyerges, M.; Rudas, M.; Bitter, I.; Toke, L. Tetrahedron 1997, 53, 3269-3280. (c) Gomez-Monterrey, I. M.; Campiglia, P., Bertamino, A.; Aquino, C.; Mazzoni, O.; Diurno, M. V.; Iacovino, R.; Saviano, M.; Sala, M.; Novellino, E.; Grieco, P. Eur. J. Org. Chem. 2008, 1983-1992. (d) Hili, R.; Yudin, A. K. J. Am. Chem. Soc. 2006,128, 14772-14773. 